\title{
Evidências de Validade da Escala de Engajamento na Carreira no Contexto Militar
}

\author{
Alexandra de Oliveira Rodrigues-Marçulo ${ }^{1}$ \\ ${ }^{1}$ Instituto Federal de Roraima, RR, Brasil.
}

\author{
Maria Cristina Ferreira ${ }^{2}$ \\ ${ }^{2}$ Universidade Salgado de Oliveira, RJ, Brasil.
}

Larissa Maria David Gabardo-Martins ${ }^{2}$

${ }^{2}$ Universidade Salgado de Oliveira, RJ, Brasil.

\begin{abstract}
Resumo: O engajamento na carreira associa-se a comportamentos proativos capazes de intensificar o desenvolvimento da carreira. Este estudo buscou reunir evidências de validade de estrutura interna da Escala de Engajamento na Carreira e de suas relações com variáveis externas, em contexto militar. A amostra foi composta por 467 militares do Exército Brasileiro, de ambos os sexos (94,4\% do sexo masculino), majoritariamente pertencentes à linha bélica $(82,4 \%)$ e provenientes de diferentes estados, com destaque para o Rio de Janeiro (39,2\%). Os participantes responderam a escalas de engajamento na carreira, adaptabilidade de carreira, autoeficácia geral percebida, satisfação com a carreira e percepção atual do desenvolvimento profissional, de forma on-line. As análises fatoriais confirmatórias evidenciaram que o modelo de dois fatores de $1^{\text {a }}$ ordem com um fator de $2^{a}$ ordem apresentou os melhores índices de ajustes, diferindo do modelo original do instrumento. A Escala de Engajamento na Carreira obteve correlação significativa e positiva alta com a adaptabilidade de carreira e significativa e positiva moderada com a autoeficácia, a satisfação com a carreira e o desenvolvimento profissional. Concluiu-se que as evidências de validade obtidas possibilitam o uso futuro da escala na avaliação do engajamento na carreira no contexto militar.
\end{abstract}

Palavras-chave: Psicologia militar, Adaptação de escala, Validade estatística, Avaliação psicológica.

\section{Validity Evidence of the Career Engagement Scale in the Military Context}

\begin{abstract}
Career engagement is associated with proactive behaviors capable of enhancing career development. The study sought to gather evidence of the internal structure validity of the Career Engagement Scale and its relations to external variables in a military context. The sample consisted of 467 soldiers of the Brazilian Army, of both sexes (94.4\% male), belonging mainly to the war line (82.4\%) and coming from different states, with emphasis on Rio de Janeiro (39.2\%). The participants answered on-line the scales for measuring career engagement, career adaptability, perceived general self-efficacy, satisfaction with the career and current perception of professional development. The confirmatory factor analyses showed that the model of two factors of 1st order with a factor of 2nd order was the one that presented the best fit indexes, differing from the original model of the instrument. The Career Engagement Scale obtained a significant and positive high correlation with career adaptability and a significant and positive moderate correlation with self-efficacy, career satisfaction, and professional development. We concluded that the validity evidence obtained allow the future use of the scale for evaluating career engagement in the military context.
\end{abstract}

Keywords: Military psychology, Scale adaptation, Statistical validity, Psychological assessment. 


\title{
Evidencias de Validez de la Escala de Compromiso Profesional en el Contexto Militar
}

\begin{abstract}
Resumen: El compromiso profesional se asocia con comportamientos proactivos capaces de mejorar el desarrollo profesional. El estudio buscó recolectar evidencias de validez de la estructura interna de la Escala de Compromiso Profesional y de sus relaciones con variables externas en un contexto militar. La muestra estaba compuesta por 467 soldados del Ejército Brasileño, de ambos sexos (94,4\% hombres), en su mayoría pertenecientes a la línea de guerra (82,4\%), provenientes de diferentes estados, con énfasis en Río de Janeiro (39,2\%). Los participantes respondieron a las escalas de compromiso profesional, adaptabilidad profesional, autoeficacia general percibida, satisfacción con la carrera y percepción actual del desarrollo profesional en línea. Los análisis factoriales confirmatorios mostraron que el modelo de dos factores de ler orden con un factor de 2do orden fue el que presentó mejores índices de ajustes, diferenciándose del modelo original del instrumento. La Escala de Compromiso Profesional obtuvo una alta correlación significativa y positiva con la adaptabilidad a la carrera y una correlación significativa y positiva moderada con la autoeficacia, la satisfacción profesional y el desarrollo profesional. Se concluyó que las evidencias de validez de la escala permiten su uso futuro en la evaluación del compromiso profesional, en el contexto militar.
\end{abstract}

Palabras clave: Psicología militar, Adaptación de escalas, Validez estadística, Evaluación psicológica.

\section{Introdução}

As pesquisas têm evidenciado que os empregados que não veem futuro no desempenho de suas funções laborais costumam mostrar-se insatisfeitos e vulneráveis a oportunidades externas, bem como desenvolver afetos negativos em relação a seu trabalho (Sinha, 2020). Ao contrário, quando os locais de trabalho apresentam uma forte cultura de desenvolvimento de carreira, aqui considerada como o conjunto de atividades que definem os papéis profissionais ocupados pelo indivíduo ao longo de sua vida (Pickerell, 2013), eles estimulam o engajamento na carreira desses profissionais (Neault \& Pickerell, 2011; Pickerell \& Neault, 2012).

$\mathrm{O}$ construto engajamento na carreira tem, portanto, despertado cada vez maior interesse das organizações que estão em busca de profissionais produtivos e comprometidos, na medida em que tal construto aumenta a produtividade e, consequentemente, os retornos organizacionais positivos (Neault \& Pickerell, 2011). Contudo, as pesquisas sobre tal construto têm se tornado um grande desafio, em razão da inexistência de uma definição única para ele e pelo fato de ser por vezes confundido com o engajamento no trabalho (Pickerell \& Neault, 2012, 2016).
Ressalte-se, porém, que o engajamento no trabalho consiste em um estado psicológico estável, de natureza afetivo-cognitiva, que se manifesta na dedicação (envolvimento), vigor (níveis elevados de energia) e absorção (elevada concentração) no trabalho (Schaufeli, Salanova, González-Romá, \& Bakker, 2002). O engajamento na carreira, por sua vez, diz respeito à adoção de comportamentos proativos dirigidos ao desenvolvimento da própria carreira (Hirschi, Freund, \& Herrmann, 2014).

Nesse sentido, o engajamento na carreira manifesta-se por meio de diversos comportamentos proativos, como o planejamento de carreira, a autoexploração de carreira, a exploração do ambiente de carreira, o estabelecimento de redes de relacionamentos e o desenvolvimento voluntário do capital humano/habilidades, que objetivam intensificar o desenvolvimento da carreira (Fay \& Frese, 2001; Hirschi et al., 2014; Rasdi, Ismail, \& Garavan, 2011), ao preparar os indivíduos para escolhas pautadas no alcance das próprias metas de trabalho no século XXI (Chan, 2007; Porfeli, 2008; Savickas, 1999; Zikic \& Hall, 2009). Logo, o engajamento na carreira aumenta os sentimentos positivos e a satisfação com as escolhas 
realizadas ao longo do desenvolvimento da carreira (Le, Jiang, \& Nielsen, 2016; Mcllveen \& Perera, 2015; Neault \& Pickerell, 2011).

No que diz respeito à avaliação do engajamento na carreira, Hirschi et al. (2014) apontam que, durante muito tempo, as medidas na área de gestão de carreira constituíram-se em diversos instrumentos unidimensionais, destinados a avaliar diferentes comportamentos proativos associados ao desenvolvimento da carreira, inexistindo, dessa forma, instrumentos que operacionalizassem os comportamentos proativos de carreira de forma global. Fundamentando-se nessas considerações, os autores introduziram a expressão engajamento na carreira, bem como desenvolveram e apresentaram evidências de validade de uma escala destinada a avaliar o referido construto, mediante a realização de seis estudos, conduzidos com seis amostras distintas.

Em um primeiro estudo, Hirschi et al. (2014) empreenderam uma revisão de literatura e identificaram seis comportamentos específicos de carreira capazes de representar adequadamente as diferentes manifestações dos indivíduos ativamente engajados no desenvolvimento de sua carreira. São eles: autoexploração de carreira, ou seja, autorreflexão sobre as habilidades e interesses de carreira atual ou futura (Stumpf, Colarelli, \& Hartman, 1983); exploração do ambiente de carreira, isto é, obtenção de informações sobre opções de carreira no ambiente externo ao indivíduo (Stumpf et al., 1983); planejamento de carreira, ou seja, compatibilização dos objetivos de carreira e habilidades individuais com as oportunidades para sua realização (Uhl-Bien, Schermerhorn Jr., \& Osborn, 2016); estabelecimento de rede de relacionamentos, mediante construção, manutenção e uso de relacionamentos para a obtenção de sucesso na carreira (Wolff \& Moser, 2009); estratégias de carreira associadas a habilidades, isto é, desenvolvimento de habilidades necessárias ao alcance de futuras posições profissionais (Gould \& Penley, 1984); e estratégias de carreira associadas à expertise, ou seja, busca de conhecimentos e experiências em áreas consideradas críticas para a carreira (Gould \& Penley, 1984).

Em seguida, Hirschi et al. (2014) revisaram diversas escalas que mensuravam esses comportamentos específicos e chegaram à versão inicial de uma escala composta por três itens para cada um dos comportamentos, que posteriormente foi submetida à apreciação de um grupo de 24 estudantes universitários alemães. Tal procedimento resultou em uma escala de nove itens, a serem respondidos em uma escala Likert de cinco pontos, variando de 1 (quase nunca) a 5 (muitas vezes), que foi aplicada a uma amostra de 146 estudantes universitários alemães (sexo feminino $=42 \%$; idade média $=23$ anos). As análises fatoriais exploratórias realizadas resultaram em uma solução de um único fator, que explicou $49,4 \%$ da variância total e obteve um Alfa de Cronbach de 0,89.

No segundo estudo, Hirschi et al. (2014) propuseram-se a examinar a estrutura fatorial e a adequação dos itens, bem como a invariância da medida segundo o gênero. Participaram deste estudo 2.027 alunos de uma universidade alemã (sexo feminino $=63,8 \%$. idade média $=23,74$ anos). Os resultados das análises fatoriais confirmatórias apontaram que o modelo de nove itens apresentou índices de ajustes satisfatórios $\left(\mathrm{SB}-x^{2}=324,79, \mathrm{~d} f=27, p<0,01 ; \mathrm{CFI}=0,96\right.$; $\mathrm{TLI}=0,94$; RMSEA $=0,07$ ) e bons índices de consistência interna $(0,88$ para amostra total). Verificou-se, ainda, a invariância da medida para os dois gêneros.

Em um terceiro estudo, Hirschi et al. (2014) objetivaram estabelecer a invariância da medida em um intervalo de seis meses e avaliar se o grau de engajamento na carreira mudava durante a universidade. Participaram do estudo 951 estudantes universitários alemães (sexo feminino $=45 \%$; média de idade $=23,70$ ) e os resultados das análises fatoriais confirmatórias dos diferentes modelos testados confirmaram a invariância da medida ao longo do tempo, bem como a adequação do instrumento à realização de pesquisas longitudinais.

No quarto estudo, Hirschi et al. (2014) procuraram demonstrar a adequação da estrutura unifatorial da escala, em uma amostra de 290 egressos de duas universidades do norte da Alemanha (sexo feminino $=56 \%$, média de idade $=29,61$ ), que já trabalhavam em diferentes ocupações, nas áreas de administração (18\%), educação (15\%) e engenharia (11\%), dentre outras. Adicionalmente, pretenderam também avaliar o grau de invariância da medida entre esse grupo e o grupo de estudantes universitários que participou do estudo 2. Os resultados das análises fatoriais confirmatórias apontaram que a solução de um fator apresentou índices de ajuste satisfatórios também na amostra de profissionais ( $\mathrm{SB}-x^{2}=54,91$, $\mathrm{d} f=27, p<0,01$; CFI $=0,97$; TLI $=0,95$; RMSEA $=0,06$ ). Foi ainda demonstrada a invariância da medida entre alunos e trabalhadores. 
Em um quinto estudo, Hirschi et al. (2014) verificaram a validade incremental da Escala de Engajamento na Carreira em comparação aos comportamentos de gestão da carreira (planejamento de carreira, autoexploração de carreira, exploração do ambiente de carreira e rede de relacionamento), na explicação de diversas atitudes sobre o trabalho. Participaram deste estudo 681 estudantes universitários alemães (sexo feminino $=62,3 \%$; média de idade $=23,51)$ e 271 trabalhadores de cinco universidades do norte da Alemanha (sexo feminino $=62 \%$; média de idade $=29,07$ ). As análises de regressão hierárquica evidenciaram que, na amostra de estudantes, o engajamento na carreira explicou variância adicional aos comportamentos de gestão de carreira, na predição da autoeficácia de carreira $(1,7 \%$ de variância adicional; $\mathrm{p}<0,001)$ e da clareza de identidade vocacional $(0,4 \%$ de variância adicional; $\mathrm{p}<0,001)$. Já na amostra de trabalhadores, o engajamento na carreira explicou variância adicional aos comportamentos de gestão de carreira, na predição da satisfação no trabalho ( $2,8 \%$ de variância adicional; $\mathrm{p}<0,001)$ e da satisfação com a carreira (6,5\% de variância adicional; $\mathrm{p}<0,001)$.

No sexto e último estudo, de natureza longitudinal, Hirschi et al. (2014) estabeleceram a utilidade incremental da medida de engajamento na carreira em comparação aos comportamentos de gestão de carreira, na predição da satisfação no trabalho e da satisfação com a carreira. Os dados foram coletados em dois tempos, sendo que em $\mathrm{T} 1 \mathrm{a}$ amostra foi composta por alunos de uma universidade alemã que estavam no último semestre e em T2 (seis meses após T1), a amostra foi composta pelos mesmos estudantes de T1 que, na ocasião, já estavam trabalhando. Desse modo, a amostra final com os dados de T1 e T2 ficou composta por 144 participantes (sexo feminino $=0 \%$; média de idade $=27,17$ ). As análises de regressão hierárquica demonstraram que o engajamento na carreira explicou variância adicional (na comparação com os comportamentos de gestão de carreira) na predição da satisfação no trabalho $(3,7$ \% de variância adicional; $\mathrm{p}<0,013$ ) e na satisfação com a carreira $(8,1 \%$ de variância adicional; $\mathrm{p}<0,001)$.

Mais recentemente, Marques, Oliveira e MeloSilva (2019) adaptaram a Escala de Engajamento na Carreira (Hirschi et al., 2014), que denominaram EEC-Br, para estudantes universitários brasileiros, e obtiveram evidências iniciais de sua validade convergente. Inicialmente, a adequação dos itens ao contexto cultural brasileiro foi avaliada por três juízas, experts na área de Orientação Profissional e Construção de Medidas, mediante a análise de três dimensões: clareza de linguagem (nitidez da linguagem utilizada nos itens, considerando a população respondente), pertinência prática (se o item foi elaborado de forma a avaliar o conceito de interesse) e relevância teórica (grau de associação entre o item e a teoria). Em seguida o instrumento foi avaliado por dez representantes do público-alvo.

Após a realização das alterações sugeridas, o instrumento foi aplicado a uma amostra de 424 estudantes universitários, com o objetivo de avaliar sua estrutura interna. A amostra foi dividida em duas subamostras independentes, sendo que na primeira (200 estudantes) os resultados das análises fatoriais confirmatórias apontaram que os indicadores de ajustamento do modelo não atingiram os critérios recomendados $(\chi 2 /(27)=151,083, p<0,001, \mathrm{~N}=200 ; \chi 2$ / $\mathrm{gl}=5,596 ; \mathrm{CFI}=0,843$; RMSEA = 0,152). Sendo assim, foi necessário reespecificá-lo, quando então ele passou a apresentar parâmetros de ajustamento dentro de valores adequados $\left(X^{2} /(22)=59,510 ; p<0,001\right.$, $\mathrm{N}=200 ; \chi 2 / \mathrm{gl}=2,705 ; \mathrm{CFI}=0,953 ; \mathrm{RMSEA}=0,093$ ) (Marques et al., 2019).

Na segunda subamostra (224 estudantes), novas análises fatoriais confirmatórias foram conduzidas para examinar a estabilidade do ajuste do modelo e seus resultados indicaram um ajuste aceitável $(\chi 2 /(21)=47,865 ; p<0,001, \mathrm{~N}=224 ; \chi 2 / \mathrm{gl}=2,185 ;$ $\mathrm{CFI}=0,975$; RMSEA $=0,073$ ). Além disso, a EEC-Br foi correlacionada com as versões brasileiras das Escalas de Identidade Profissional (EIP-Br) $(r=0,42$; $p<0,01)$ e de Clareza de Identidade Profissional (ECIP-Br) $(r=0,44 ; p<0,01)$. As correlações positivas e significativas obtidas constituíram-se, portanto, em evidências iniciais sobre a validade convergente da escala em estudantes universitários brasileiros (Marques et al., 2019).

Em que pese o fato de Hirschi et al. (2014), no estudo original de construção da Escala de Engajamento na Carreira, terem reunido evidências de validade da escala em amostras de estudantes universitários e de trabalhadores, o estudo de adaptação brasileira da escala (Marques et al., 2019) reuniu evidências de sua validade apenas com estudantes universitários. Contudo, o estudo do engajamento em comportamentos proativos de carreira mostra-se 
relevante não apenas entre estudantes universitários, mas também entre pessoas que já trabalham, visto que o gerenciamento da própria carreira consiste em um processo que emerge na universidade, mas acompanha o indivíduo ao longo de toda a sua vida profissional (Hirschi et al., 2014). Por conseguinte, o desenvolvimento de carreira constitui fator fundamental para que a organização possa contar com uma força de trabalho competitiva (Wong, Rasdi, Samah, \& Wahat, 2017).

De particular interesse para o presente trabalho é o engajamento na carreira militar, em função de que, nesse contexto, a gestão de carreiras ancora-se nas exigências de cada função, mas implica também a identificação do potencial de desenvolvimento profissional dos militares, bem como de suas próprias aspirações e de sua capacidade de adaptação às novas funções. O objetivo é, portanto, orientar sua formação continuada, oferecendo-lhes preparação adequada ao desempenho de cargos de maior complexidade e responsabilidade, à medida que progridem na hierarquia militar (Santos, 2013). Desse modo, a avaliação do engajamento na carreira no contexto militar poderá funcionar como mais uma ferramenta que, juntamente com as demais avaliações de habilidades e potencialidades, poderão contribuir para dar suporte ao processo de formação continuada e de progressão na carreira militar.

No entanto, levantamento realizado em bases de dados nacionais e internacionais (Psychinfo, Scientific Eletronic Library Online [SciELO], Portal de Periódicos Eletrônicos de Psicologia [PePSIC] e Banco de teses da Capes), no mês de agosto de 2019, com os descritores "engajamento na carreira no contexto militar"; "engajamento na carreira nas forças armadas"; "engajamento em contexto militar"; e "engajamento na carreira militar", em português e em inglês, usando-se os conectores e/and e ou/or, apontou a inexistência, até o momento, de estudos destinados a buscar evidências de validade da Escala de Engajamento na Carreira em amostras de militares. Justifica-se, assim, a realização de estudos futuros sobre o engajamento na carreira em militares de carreira da ativa, que poderão fornecer subsídios valiosos à avaliação e redirecionamento das práticas de desenvolvimento da carreira militar, de modo a torná-las mais adequadas a cada indivíduo em particular e permitir, assim, melhor interação entre ele e seu ambiente institucional.
Fundamentando-se, portanto, nos fatos de a avaliação do engajamento na carreira ser relevante ao contexto militar e de inexistirem estudos com esse foco em amostras brasileiras, o presente estudo teve como objetivo geral reunir evidências de validade de estrutura interna e de relações com variáveis externas da Escala de Engajamento na Carreira (Career Engagement Scale) (Hirschi et al., 2014), na versão adaptada por Marques et al. (2019), em militares brasileiros, como forma de preencher tais lacunas. Em relação aos objetivos específicos, o estudo pretendeu testar quatro hipóteses, acerca das relações do engajamento na carreira com as seguintes variáveis externas: adaptabilidade de carreira (Savickas, 2013), autoeficácia geral percebida (Zhou, 2015), satisfação com a carreira (Spurk, Abele, \& Volmer, 2011) e desenvolvimento profissional (Mourão, Porto, \& PuentePalacios, 2014b), em função de tais construtos terem apresentado relações com os comportamentos de carreira em estudos anteriores (Hirschi et al., 2014; Nilforooshan \& Salimi, 2016).

A adaptabilidade de carreira denota a prontidão à mudança e o ajustamento a circunstâncias novas ou modificadas (Savickas, 2013). Estudo realizado por Nilforooshan e Salimi (2016) observou uma relação positiva elevada entre o engajamento na carreira e a adaptabilidade de carreira. Sendo assim, hipotetizou-se uma associação positiva alta entre o engajamento na carreira e adaptabilidade de carreira no contexto militar $(\mathrm{H} 1)$.

A autoeficácia geral percebida consiste na crença que o indivíduo tem sobre sua capacidade de realizar com sucesso suas atividades profissionais (Zhou, 2015). Estudo realizado por Hirschi et al. (2014) apontou uma correlação positiva moderada entre as crenças de autoeficácia na carreira e o engajamento na carreira. Desse modo, formulou-se a hipótese de que existiria uma correlação positiva moderada entre o engajamento na carreira e a autoeficácia geral percebida no contexto militar $(\mathrm{H} 2)$.

A satisfação com a carreira expressa-se por meio de avaliações que os indivíduos têm acerca de seu progresso individual e de suas perspectivas futuras de evolução e sucesso na carreira (Hofmans, Dries, \& Pepermans, 2008; Spurk et al., 2011). Os estudos de Hall, Waddell, Donner e Wheeler (2004) e de Hirschi et al. (2014) demonstraram que pessoas engajadas em sua carreira exibem maiores níveis de satisfação com a carreira. Desse modo, foi hipotetizado que o 
engajamento na carreira apresentaria uma correlação positiva moderada com a satisfação com a carreira no contexto militar (H3).

O desenvolvimento profissional consiste no processo de aquisição e aperfeiçoamento de conhecimentos, habilidades e atitudes que favorecem o desempenho no trabalho e o avanço individual na carreira (Mourão et al., 2014b). Estudos empíricos têm verificado que as oportunidades de desenvolvimento profissional impactam positivamente o engajamento dos empregados com sua carreira (Hirschi et al., 2014; Shafer, 2010). Assim, formulou-se a hipótese de que haveria uma correlação positiva moderada entre essas variáveis, no contexto militar (H4).

\section{Método}

\section{Participantes}

A amostra foi composta por 467 militares do Exército Brasileiro, de ambos os sexos (94,4\% do sexo masculino), pertencentes a diferentes quadros, armas e serviços, mas foi constituída, em sua maioria, por majores $(32,0 \%)$, seguidos de capitães $(16,3 \%)$ e primeiro-sargentos $(13,1 \%)$, pertencentes principalmente à linha bélica $(82,4 \%)$, e provenientes de todas as regiões do país, com predominância do Rio de Janeiro (39,2\%), seguido do Rio Grande do Sul (18,8 \%) e Minas Gerais $(12,4 \%)$. Os grupos etários foram, na maior parte, de 31 a 40 anos (43,7\%) e de 41 a 50 anos $(40,3 \%)$. Quanto ao estado civil, a maioria era casada $(72,2 \%)$ e tinha filhos $(77,1 \%)$. No que tange à escolaridade, grande parte da amostra tinha ensino superior completo e pós-graduação $(59,1 \%$ ), bem como tempo de efetivo serviço acima de 20 anos (54,6\%), seguido de 16 a 20 anos (21,0\%). Para inclusão no estudo, utilizou-se os critérios de o participante ser militar da ativa de carreira e de ter pelo menos um ano de serviço, após a formação nas escolas militares. Todos os demais militares que não se enquadravam nesses critérios foram, portanto, excluídos.

\section{Instrumentos}

Para mensurar o engajamento na carreira utilizou-se a Escala de Engajamento na Carreira (Career Engagement Scale) (Hirschi et al., 2014), adaptada ao contexto brasileiro por Marques et al. (2019). O instrumento é unidimensional e composto por nove itens, a serem respondidos em uma escala Likert de cinco pontos, variando de 1 (não muito) a 5 (sempre). Exemplo de item: "Assumiu responsabilidade ou posições que poderão ajudá-lo a progredir na sua carreira". A consistência interna da escala, no estudo de Marques et al. (2019), avaliada pelo Alfa de Cronbach, foi de 0,89 .

Para mensurar a adaptabilidade de carreira utilizou-se a Escala de Adaptabilidade de Carreira (Career Adapt-Abilities Scale [CAAS]), de Savickas e Porfeli (2012), na versão brasileira revisada de Audibert e Teixeira (2015). O instrumento é composto por 24 itens, distribuídos em quatro dimensões: preocupação (6 itens), controle (6 itens), curiosidade (6 itens) e confiança (6 itens), a serem respondidos em uma escala Likert de cinco pontos, variando de 1 (desenvolvi pouco ou nada) a 5 (desenvolvi extremamente bem). Exemplo de item: "Atualmente, exploro as opções antes de fazer uma escolha". A consistência interna das subescalas, no estudo de Audibert e Teixeira (2015), avaliada pelo Alfa de Cronbach, foi igual a: preocupação - 0,88 ; controle $-0,83$; curiosidade-0,88; confiança-0,89.

Para mensurar a autoeficácia geral percebida utilizou-se a Escala de Autoeficácia Geral Percebida (Nunes, Schwarzer, \& Jerusalem, 1999), na versão brasileira de Sbicigo, Teixeira, Dias e Dell'Aglio (2012). O instrumento é unidimensional e composto por dez itens, a serem respondidos em uma escala Likert de quatro pontos, variando de 1 (não é verdade a meu respeito) a 4 (é totalmente verdade a meu respeito). Exemplo de item: "Tenho facilidade para persistir em minhas intenções e alcançar meus objetivos". A consistência interna da escala, no estudo de Sbicigo et al. (2012), avaliada pelo Alfa de Cronbach, foi de 0,85.

Para mensurar a satisfação com a carreira utilizou-se a Escala de Satisfação com a Carreira (Greenhaus, Parasuraman, \& Wormley, 1990), na versão adaptada por Seibert, Crant e Kraimer (1999). O instrumento é unidimensional e composto por cinco itens, a serem respondidos em uma escala Likert de cinco pontos, variando de 1 (discordo totalmente) a 5 (concordo totalmente). Exemplo de item: "Estou satisfeito com o sucesso que alcancei na minha carreira”. A consistência interna da escala, no estudo de Seibert et al. (1999), avaliada pelo Alfa de Cronbach, foi de 0,88.

Para mensurar o desenvolvimento profissional utilizou-se uma versão reduzida da Escala de Percepção Atual do Desenvolvimento Profissional (EPADP) (Mourão et al., 2014b). O instrumento é 
unidimensional e composto por quatro itens, a serem respondidos em uma escala Likert de cinco pontos, variando de 1 (discordo fortemente) a 5 (concordo fortemente). Exemplo de item: "Atualmente domino todas as habilidades necessárias para a realização do meu trabalho". A consistência interna da escala, no estudo de estudo de Mourão et al. (2014b), avaliada pelo Alfa de Cronbach, foi de 0,86.

\section{Procedimentos de coleta de dados e cuidados éticos}

Inicialmente, a pesquisa foi submetida ao Comitê de Ética em Pesquisa da instituição dos autores e aprovado sob o número CAAE 86574318.2.0000,5289. Um ofício da instituição foi enviado à Diretoria de Educação e Cultura do Exército (Decex), para obtenção de autorização para a aplicação da pesquisa na instituição.

Os convites à participação foram enviados por meio de documento oficial interno (Diex) endereçado a militares no âmbito do Decex (Diretorias/ Centro, Estabelecimentos de Ensino, Organizações Militares subordinadas e/ou vinculadas) e a coleta de dados foi realizada de forma on-line, a partir da ferramenta Google Forms, disponibilizada nas páginas do Decex (internet e Intranet). Vale ressaltar que, na aplicação, houve a concordância prévia sobre a participação na pesquisa, mediante o preenchimento do Termo de Consentimento Livre e Esclarecido (TCLE), que constava como parte inicial do instrumento de coleta de dados. As escalas foram todas aplicadas na mesma ordem utilizada na seção de descrição dos instrumentos e somente as preenchidas em sua totalidade foram consideradas nas análises subsequentes.

\section{Procedimentos de análise de dados}

Com o intuito de verificar a estrutura interna da Escala de Engajamento na Carreira, a amostra foi dividida em duas subamostras. Inicialmente, na primeira subamostra, realizou-se a Análise Fatorial Exploratória (AFE), no software R (R Core Team, 2017), no pacote Psych (Revelle, 2016). Em seguida, na outra subamostra, foi realizada a Análise Fatorial Confirmatória no software R (R Core Team, 2017), no pacote Lavan (Rosseell, 2012), com o objetivo de verificar se a estrutura obtida na AFE apresentaria índices adequados de ajuste. Por fim, o teste de
Shapiro Wilk apontou a não normalidade dos dados. Nesse sentido, as hipóteses de relação entre as variáveis foram testadas por meio do cálculo das correlações entre elas, adotando-se o rho de Spearman e considerando os seguintes critérios: correlações baixas $(0,10$ a 0,29$)$, moderadas $(0,30$ a 0,49$)$ e altas (acima de 0,50) (Miles \& Shevlin, 2001).

\section{Resultados}

O resultado do Kaiser-Meyer-Olkin (KMO) foi de 0,91, numa demonstração de que a amostra era adequada à realização da AFE. O teste de Bartlett foi significativo (qui-quadrado $=2463,81 ; p<0,001$ ), indicando que a matriz de correlações entre os itens também permitia a realização da AFE. A análise do scree plot sugeriu que a escala seria composta por dois fatores, enquanto a análise paralela indicou que o instrumento seria composto de três fatores. Nesse sentido, foi inicialmente realizada uma AFE com a fixação de três fatores a serem extraídos, sendo que os resultados apontaram que dois fatores ficaram compostos por apenas um item, que tinha também apresentado carga fatorial elevada em outro fator. Em seguida, foi realizada uma outra AFE com a fixação de dois fatores. Os resultados obtidos indicaram que todos os itens foram retidos em seus respectivos fatores e apresentaram cargas fatoriais adequadas, conforme pode ser visto na Tabela 1 . $\mathrm{Na}$ análise semântica dos itens, os fatores foram rotulados como: Fator 1 (itens de 1 a 4) - planejamento de carreira; Fator 2 (itens de 5 a 9) - busca de oportunidade profissional.

Considerando os resultados encontrados na AFE e a estrutura unifatorial do modelo original do instrumento, foram testados os seguintes modelos na Análise Fatorial Confirmatória: um fator; dois fatores de primeira ordem; dois fatores de primeira ordem com um fator de segunda ordem. Os resultados evidenciaram que o modelo de dois fatores de $1^{\text {a }}$ ordem com um fator de $2^{\text {a }}$ ordem apresentou os melhores índices de ajustes (Tabela 2), razão pela qual ele foi escolhido como o que melhor representava a estrutura do instrumento.

Vale ressaltar, ainda, que os itens em geral obtiveram cargas fatoriais elevadas $(M=0,76)$, numa demonstração de que eles podem ser explicados por seus respectivos fatores. A Figura 1 traz a representação gráfica do modelo final retido, com suas cargas fatoriais padronizadas. 
Tabela 1

Itens e cargas fatoriais da Escala de Engajamento na Carreira.

\begin{tabular}{cc}
\hline Itens & Fator 1 \\
\hline 1. Procurou ativamente planejar seu futuro & 0,80 \\
2. Realizou atividades importantes para alcançar seus objetivos de carreira 2 & 0,74 \\
3. Preocupou-se com o desenvolvimento da sua carreira & 0,95 \\
4. Construiu planos e definiu objetivos para sua carreira & 0,88 \\
5. Refletiu, sinceramente, sobre seus valores pessoais, & 0,39 \\
suas habilidades e suas fraquezas & 0,55 \\
6. Coletou informações sobre empregadores, & 0,82 \\
de trabalho em sua área de interesse & 0,79 \\
7. Estabeleceu contato com pessoas que poderiam colaborar \\
no desenvolvimento de sua carreira
\end{tabular}

Tabela 2

Análise fatorial confirmatória da Escala de Engajamento na Carreira.

\begin{tabular}{ccccc}
\hline Modelos testados & $\mathrm{x}^{2}(\mathrm{gl})$ & CFI & TLI & RMSEA \\
\hline Um fator & $166,65(27)$ & 0,85 & 0,80 & 0,11 \\
Dois fatores de 1a ordem & $174,15(26)$ & 0,84 & 0,78 & 0,11 \\
Dois fatores de $1^{\text {a }}$ ordem e um fator de 2 ${ }^{\text {a }}$ ordem & $166,36(27)$ & 0,98 & 0,98 & 0,10 \\
\hline
\end{tabular}

Legenda: $\mathrm{x}^{2}$ = qui-quadrado; $\mathrm{gl}$ = graus de liberdade; $\mathrm{TLI}$ = Tucker Lewis Index; CFI = Comparative Fit Index; RMSEA = Root Mean Square Error of Approximation.

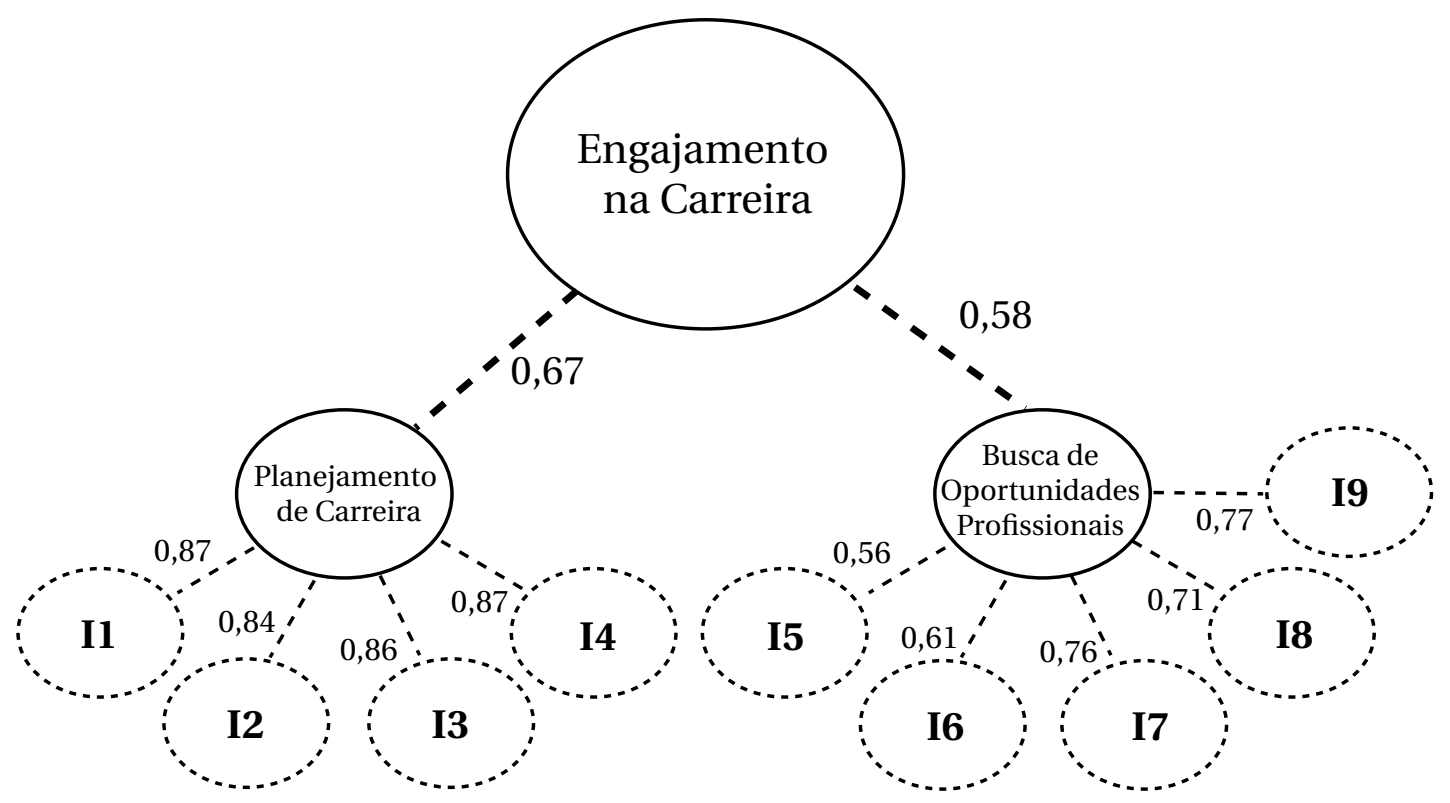

Figura 1

Modelo final da Escala de Engajamento na Carreira, com as cargas fatoriais padronizadas. 
$\mathrm{Na}$ análise de confiabilidade, os fatores planejamento de carreira e busca de oportunidade profissional apresentaram, respectivamente, Alfas de Cronbach iguais a 0,92 e 0,81. O fator geral apresentou um índice igual a 0,90 . As demais escalas adotadas no estudo também apresentaram bons índices de confiabilidade (Tabela 3). Na correlação da escala de engajamento na carreira com variáveis externas, os resultados indicaram que, em geral, ela apresentou: correlações significativas $(\mathrm{p}<0,01)$ e positivas altas com a dimensão geral e com as dimensões de preocupação e curiosidade, da escala de adaptabilidade de carreira; e correlações significativas $(\mathrm{p}<0,01)$ e positivas moderadas com as dimensões de controle e de confiança da escala de adaptabilidade de carreira, bem como com as escalas de autoeficácia geral percebida, satisfação com a carreira e desenvolvimento profissional, como pode ser visto na Tabela 3.

Tabela 3

Médias, Desvios-Padrão, Coeficientes de Correlação entre as Dimensões da Escala de Engajamento na Carreira e as demais Escalas do Estudo e Índices de Consistência Interna das Escalas.

\begin{tabular}{cccccccccccc}
\hline & Média & $\begin{array}{c}\text { Desvio } \\
\text { padrão }\end{array}$ & 1 & 2 & 3 & 4 & 5 & 6 & 7 & 8 & 9 \\
\hline & & & & & & & & & & & \\
$1 \mathrm{EC}$ & 3,61 & 0,83 & $(0,90)$ & - & - & - & - & - & - & - & - \\
$2 \mathrm{AD}$ & 3,89 & 0,63 & $0,58^{* *}$ & $(0,95)$ & - & - & - & - & - & - & - \\
$3 \mathrm{AP}$ & 3,69 & 0,81 & $0,56^{* *}$ & $0,84^{* *}$ & $(0,90)$ & - & - & - & - & - & - \\
$4 \mathrm{ACnt}$ & 4,09 & 0,67 & $0,40^{* *}$ & $0,82^{* *}$ & $0,57^{* *}$ & $(0,84)$ & - & - & - & - & - \\
$5 \mathrm{ACrd}$ & 3,66 & 0,74 & $0,52^{* *}$ & $0,88^{* *}$ & $0,68^{* *}$ & $0,62^{* *}$ & $(0,88)$ & - & - & - & - \\
$6 \mathrm{ACnf}$ & 4,13 & 0,70 & $0,45^{* *}$ & $0,81^{* *}$ & $0,54^{* *}$ & $0,62^{* *}$ & $0,69^{* *}$ & $(0,91)$ & - & - & - \\
$7 \mathrm{AE}$ & 3,29 & 0,45 & $0,36^{* *}$ & $0,54^{* *}$ & $0,37^{* *}$ & $0,51^{* *}$ & $0,45^{* *}$ & $0,51^{* *}$ & $(0,88)$ & - & - \\
$8 \mathrm{SC}$ & 3,63 & 0,84 & $0,41^{* *}$ & $0,43^{* *}$ & $0,40^{* *}$ & $0,40^{* *}$ & $0,34^{* *}$ & $0,30^{* *}$ & $0,34^{* *}$ & $(0,88)$ & - \\
$9 \mathrm{DP}$ & 4,07 & 0,64 & $0,42^{* *}$ & $0,41^{* *}$ & $0,30^{* *}$ & $0,41^{* *}$ & $0,30^{* *}$ & $0,41^{* *}$ & $0,49^{* *}$ & $0,35^{* *}$ & $(0,81)$ \\
\hline
\end{tabular}

${ }^{* *} p<0,01$.

Nota: A diagonal principal apresenta os valores dos índices de confiabilidade (Alfa de Cronbach) das escalas.

Legenda: $\mathrm{EC}$ = engajamento na carreira; $\mathrm{AD}$ = adaptabilidade de carreira; $\mathrm{AP}$ = adaptabilidade/preocupação; $\mathrm{ACnt}=$ adaptabilidade/controle; ACrd = adaptabilidade/curiosidade; $\mathrm{ACnf}=$ adaptabilidade/confiança; $\mathrm{AE}=$ Autoeficácia geral percebida); $\mathrm{SC}$ = satisfação na carreira; $\mathrm{DP}$ = desenvolvimento profissional.

\section{Discussão}

O presente estudo teve como objetivo geral reunir evidências de validade de estrutura interna e de relações com variáveis externas da Escala de Engajamento na Carreira (EEC-Br), na versão adaptada ao contexto de estudantes universitários brasileiros por Marques et al. (2019), em uma amostra de militares. Os resultados das Análises Fatoriais Exploratórias e Confirmatórias evidenciaram que o modelo de dois fatores de $1^{\mathrm{a}}$ ordem (planejamento de carreira e busca de oportunidade profissional) com um fator de $2^{\text {a }}$ ordem (engajamento na carreira) foi o que apresentou os melhores índices de ajuste. Tais resultados diferem do modelo original da escala proposta por Hirschi et al. (2014) e da versão adaptada por Marques et al. (2019) (EEC-Br), visto que em tais estudos a versão final da escala era de natureza unifatorial.
Tais diferenças podem se dever ao fato de que a presente amostra foi de militares de carreira da ativa, com tempo de experiência profissional acima de 20 anos. Nesse sentido, ela difere das amostras de Hirschi et al. (2014) e Marques et al. (2019), que desenvolveram seus estudos majoritariamente em amostras de estudantes universitários, embora alguns já estivessem em processo de transição da universidade para o mercado de trabalho ou já fossem trabalhadores. Assim, na fase da carreira em que os participantes do presente estudo se encontravam, eles são direcionados a tomar decisões quanto aos rumos de suas carreiras (Santos, 2013). Desse modo, eles precisam fazer escolhas relacionadas a certas especializações (como fazer cursos de altos estudos ou voltar-se à área de ensino), visto que, no campo operacional, as oportunidades de especializações diminuem, em virtude 
de fatores como a idade e o vigor físico. Em outras palavras, os militares são solicitados a planejar suas carreiras de forma a buscar novas oportunidades, que os levarão aos mais altos postos da carreira, a exemplo do generalato (Santos, 2013), o que os difere dos estudantes universitários, cujas preocupações em relação à carreira voltam-se sobretudo para a entrada no mundo do trabalho (Monteiro, Sobrosa, Dalagasperina, \& Dias, 2016).

Os índices de consistência interna da escala, calculados por meio do Alfa de Cronbach, foram muito bons para o fator geral $(\alpha=0,90)$ e se assemelham aos estudos de Hirschi et al. (2014) e de Marques et al. (2019), que obtiveram, respectivamente, índices de 0,87 e 0,90 para a escala. Tais resultados apontam, portanto, que o instrumento tem apresentado precisão adequada em diferentes contextos. Desse modo, ele se revelou também uma medida útil à mensuração do engajamento na carreira de militares, de forma fidedigna.

No que diz respeito às correlações da EEC-Br com variáveis externas, verificou-se a existência de uma correlação positiva alta do engajamento na carreira com a adaptabilidade de carreira e algumas de suas dimensões, confirmando, assim, a hipótese 1 do presente estudo. Tal resultado converge com o estudo de Nilforooshan e Salimi (2016), que também observaram correlações dessa natureza entre tais construtos. Os resultados mostraram, portanto, que à medida que aumenta o engajamento na carreira, aumenta também a adaptação dos indivíduos ao longo do desenvolvimento de sua carreira, mediante um processo dinâmico que retroalimenta os comportamentos proativos específicos ao contexto de carreira (Nilforooshan \& Salimi, 2016). Além disso, o engajamento na carreira torna o indivíduo mais predisposto a lidar com as mudanças imprevisíveis ocorridas em suas condições de trabalho e a elas se adaptarem (Savickas, 2013).

$\mathrm{Na}$ análise das relações entre a EEC-Br e a autoeficácia geral percebida, os resultados apontaram para uma correlação positiva moderada, o que confirma totalmente a hipótese 2 . Tais resultados assemelham-se aos diferentes estudos (Creed, Patton, \& Prideaux, 2007; Hirschi et al., 2014; Rogers, Creed, \& Glendon, 2008) que também observaram correlações positivas moderadas entre esses construtos, numa indicação de que os indivíduos que apresentam mais comportamentos de exploração e de planejamento de sua carreira tendem a ter maiores crenças de autoeficácia geral percebida. Tal resultado pode se dever ao fato de que indivíduos com maiores níveis de engajamento costumam gerenciar melhor os comportamentos que intensificam seu desenvolvimento na carreira e, consequentemente, buscam de forma mais proativa e motivada o alcance do sucesso, o que possibilita $o$ aumento de suas crenças de autoeficácia geral e, consequentemente, o seu grau de confiança na execução de diferentes papéis profissionais (Rogers et al., 2008).

$\mathrm{Na}$ avaliação da relação entre a EEC-Br e a satisfação na carreira, os dados indicaram uma relação positiva moderada, confirmando, assim, totalmente a hipótese 3. Tais resultados assemelham-se aos estudos de Hall et al. (2004) e de Hirschi et al. (2014), que também encontraram correlações positivas moderadas entre esses construtos. Tais resultados indicam que os empregados que estão engajados em seu trabalho tendem a ter maior grau de satisfação com suas carreiras (Judge, Thoresen, Pucik, \&Welbourne, 1999). Em outras palavras, à medida que o indivíduo, no desenvolvimento de sua carreira, desenvolve e implementa estratégias de engajamento que potencializam seu grau de desempenho e produtividade, passa a vivenciar sentimentos mais positivos sobre suas experiências de trabalho, o que leva a um aumento do seu grau de satisfação com a carreira (Yap, Cukier, Holmes, \& Hannan, 2010).

No que diz respeito à relação da EEC-Br com o construto desenvolvimento profissional, os resultados apontaram para uma correlação positiva moderada, 0 que confirmou em sua totalidade a hipótese 4 . Esses achados vão ao encontro dos estudos de Hirschi et al. (2014) e Shafer (2010), que também observaram que a emissão de comportamentos proativos em relação à carreira permite um crescimento e amadurecimento dos conhecimentos, habilidades e atitudes adquiridos ao longo da vida dos trabalhadores, como resultado de ações formais e informais de aprendizagem no trabalho (Mourão et al., 2014b), ações estas que se mostraram importantes para seu desenvolvimento profissional. Sendo assim, o planejamento e a busca de oportunidades profissionais refletem-se, na prática, por meio da emissão de comportamentos adequados ao desenvolvimento profissional (Mourão, Monteiro, \& Viana, 2014a).

Os achados ora obtidos indicam, portanto, que a Escala de Engajamento na Carreira apresentou evidências de validade de estrutura interna, de relações 
com outras variáveis e de consistência interna, em amostras brasileiras pertencentes ao contexto militar. Nesse sentido, o instrumento demonstrou ser uma ferramenta útil à avaliação da percepção acerca dos comportamentos proativos dirigidos ao desenvolvimento da própria carreira entre o público militar, o que poderá se revelar como uma importante estratégia de conhecimento desse público e de tomadas de decisões relativas a sua formação continuada.

Cumpre ressaltar, porém, que a amostra foi composta tão somente por militares do Exército Brasileiro, servindo, em grande parte, no estado do Rio de Janeiro, além de se constituir em uma amostra de conveniência, razão pela qual a generalização dos resultados presentemente obtidos deve ser feita com cautela. Salienta-se, ainda, o uso de instrumentos de autorrelato, que podem afetar os resultados em virtude de os indivíduos tenderem a responder com alta desejabilidade social (Anastasi \& Urbina, 2000).

No que tange à realização de estudos futuros, seria interessante aprofundar a rede nomológica do construto engajamento na carreira em amostras de outras categorias profissionais militares, como, por exemplo, as forças auxiliares (Polícia Federal, Polícia Militar, Bombeiros, Polícia Civil, Guarda Municipal), que têm modelos de carreiras diferenciados, especialmente no que diz respeito aos planos de carreira. Tais pesquisas deveriam ser preferencialmente de natureza longitudinal, o que permitiria a obtenção de maior compreensão acerca de tais relações. A associação de variáveis sociodemográficas e profissionais como sexo, escolaridade, posto e graduação, tempo de efetivo serviço e instituição (forças armadas ou forças auxiliares) - com o engajamento na carreira, assim como a comparação de tal construto entre trabalhadores civis e militares, também poderiam ser objeto de investigações futuras.

Além disso, seria interessante também que estudos futuros averiguassem as relações do engajamento na carreira com comportamentos proativos emitidos no contexto laboral. Considerando, por exemplo, que o redesenho do trabalho associa-se a mudanças proativas nas demandas e recursos do trabalho destinadas a promover melhores ajustes entre o indivíduo e seu contexto de trabalho (Bakker \& Demerouti, 2017), é plausível supor que ele possa se constituir em uma consequência do engajamento na carreira. De modo semelhante, a cidadania organizacional, que diz respeito à emissão de comportamentos proativos e informais que visam o benefício da organização (Costa, Estivalete, \& Andrade, 2017), também poderia se constituir em uma consequência do engajamento na carreira. Em síntese, as evidências iniciais de validade ora obtidas recomendam o uso futuro da Escala de Engajamento na Carreira em investigações brasileiras destinadas a avaliar o grau de engajamento na carreira em contexto militar e suas implicações para os comportamentos proativos emitidos no contexto laboral.

\section{Referências}

Anastasi, A., \& Urbina, S. (2000). Testagem psicológica (7a ed.). Artes Médicas.

Audibert, A., \& Teixeira, M. A. P. (2015). Escala de adaptabilidade de carreira: Evidências de validade em universitários brasileiros. Revista Brasileira de Orientação Profissional, 16(1), 83-93. http://pepsic.bvsalud.org/scielo.php? script=sci_arttext\&pid=S1679-33902015000100009

Bakker, A. B., \& Demerouti, E. (2017). Job demands-resources theory: Taking stock and looking forward. Journal of Occupational Health Psychology, 22(3), 273-285. https://doi.org/10.1037/ocp0000056

Chan, D. W. (2007). Positive and negative perfectionism among Chinese gifted students in Hong Kong: Their relationships to general self-efficacy and subjective well-being. Journal for the Education of the Gifted, 31(1), 77-102. https://doi.org/10.4219/jeg-2007-512

Costa, V. F., Estivalete, V. F. B., \& Andrade, T. (2017). Comportamento de cidadania organizacional: As visões de colaboradores e de gestores de uma indústria de eletrodomésticos. Revista Eletrônica de Administração, 23(n.spe), 262-291. https:// doi.org/10.1590/1413-2311.166.62241

Creed, P. A., Patton, W., \& Prideaux, L.-A. (2007). Predicting change over time in career planning and career exploration for high school students. Journal of Adolescence, 30(3), 377-392. https://doi.org/10.1016/ j.adolescence.2006.04.003

Fay, D., \& Frese, M. (2001). The concept of personal initiative: An overview of validity studies. Human Perfomance, 14(1), 97-124. https://doi.org/10.1207/S15327043HUP1401_06 
Gould, S., \& Penley, L. E. (1984). Career strategies and salary progression: A study of their relationships in a municipal bureaucracy. Organizational Behavior and Human Performance, 34(2), 244-265. https://doi.org/ 10.1016/0030-5073(84)90006-0

Greenhaus, J. H., Parasuraman, S., \&Wormley, W. M. (1990). Effects of race on organizational experiences, job performance evaluations, and career outcomes. The Academy of Management Journal, 33(1), 64-86. https://doi.org/ $10.2307 / 256352$

Hall, M. L., Waddell, J., Donner, G., \& Wheeler, M. M. (2004). Outcomes of a career planning and development program for registered nurses. Nursing Economics, 22(5), 231- 238. https://search.proquest.com/ openview/191eb761e77cc9bec24ef4f8e73d5284/1?pq-origsite=gscholar\&cbl=30765

Hirschi, A., Freund, P. A., \& Herrmann, A. (2014). The career engagement scale: Development and validation of a measure of proactive career behaviors. Journal of Career Assessment, 22(4), 575-594. https://doi.org/ $10.1177 / 1069072713514813$

Hofmans, J., Dries, N., \& Pepermans, R. (2008). The career satisfaction scale: Response bias among men and women. Journal of Vocational Behavior, 73(3), 397-403. https://doi.org/10.1016/j.jvb.2008.08.001

Judge, T. A., Thoresen, C. J., Pucik, V., \&Welbourne, T. M. (1999). Managerial coping with organizational change: Adispositional perspective. Journal of Applied Psychology, 84(1). 107-122. https://doi.org/ 10.1037/0021-9010.84.1.107

Le, H., Jiang, Z., \& Nielsen, I. (2016). Cognitive cultural intelligence and life satisfaction of migrant workers: The roles of career engagement and social injustice. Social Indicators Research, 139(1), 237-257. https://doi.org/10.1007/ s11205-016-1393-3

Marques, B. C. S., Oliveira, M. C., \& Melo-Silva, L. L. (2019). Escala de engajamento com a carreira (EEC). In A. L. de Andrade, M. F. O. Nunes, M. Z. de Oliveira \& R. A. M. Ambiel (Eds.), Técnicas e medidas em orientação profissional e de carreira (pp. 49-60). Vetor.

Mcllveen, P., \& Perera, H. N. (2015). Career optimism mediates the effect of personality on teachers' career engagement. Journal of Career Assessment, 24(4), 623-236. https://doi.org/10.1177/1069072715616059

Miles, J. N. V., \& Shevlin, M. E. (2001). Applying regression and correlation: A guide for students and researchers. Sage.

Monteiro, J. K, Sobrosa, G. M. R., Dalagasperina, P., \& Dias, A. C. G. (2016). Desenvolvimento e planejamento de carreira em universitários: Um estudo exploratório. Revista Trabalho (En)Cena, 1(2), 145-161. https://sistemas. uft.edu.br/periodicos/index.php/encena/article/download/2879/9702/

Mourão, L., Monteiro, A. C. F., \&Viana, V. R. (2014a). A influência do desenvolvimento profissional e da identificação profissional na satisfação no trabalho. Psico, 45(2), 198-208. https://doi.org/10.15448/1980-8623.2014.2.13470

Mourão, L., Porto, J. B., \& Puente-Palacios, K. (2014b). Construção e evidências de validade de duas escalas de percepção de desenvolvimento profissional. Psico-USF, 19(1), 73-85.https://doi.org/10.1590/S1413-82712014000100008

Neault, R. A., \& Pickerell, D. A. (2011). Career engagement: Bridging career counseling and employee engagement. Journal of Employment Counseling, 48(4), 185-188. https://doi.org/10.1002/j.2161-1920.2011.tb01111.x

Nilforooshan, P., \& Salimi, S. (2016). Career adaptability as a mediator between personality and career engagement. Journal of Vocational Behavior, 94(2), 1-10. https:// doi.org/10.1016/j.jvb.2016.02.010

Nunes, R., Schwarzer, R., \& Jerusalem, M. (1999). A escala de auto-eficácia geral percepcionada. http://userpage. fu-berlin.de/ health/auto.htm

Pickerell, D. A. (2013). Examining the career engagement of Canadian career development practitioners [Tese de doutorado não publicada, Fielding Graduate University].

Pickerell, D. A., \& Neault, R. A. (2012). Where's the work? Helping career development practitioners explore their career options. Life Strategies Ltd. http://lifestrategies.ca/store/proddetail.php?prod=WherestheWork

Pickerell, D. A., \& Neault, R. A. (2016). Examining the career engagement of Canadian career development practitioners. Canadian Journal of Career Development, 15(1), 4-14. https://cjcd-rcdc.ceric.ca/index.php/cjcd/article/ view/157

Porfeli, E. J. (2008). Career exploration. In F. T. L. Leong, W. B. Walsh \& P. J. Hartung (Eds.), Career counseling (pp. 1474-1477). Sage. 
Rodrigues-Marçulo, A. de O., Ferreira, M. C., \& Gabardo-Martins, L. M. D. (2022). Validação da Escala de Engajamento na carreira.

Rasdi, R. M., Ismail, M., \& Garavan, T. N. (2011). Predicting Malaysian managers' objective and subjective career success. International Journal of Human Resource Management, 22(17), 3528-3549. https://doi.org/10.1080/ 09585192.2011.560878

R Core Team. (2017). R: A language and environment for statistical computing [Software]. $R$ Foundation for Statistical Computing. http://www.R-project.org/

Revelle, W. (2016). Psych: Procedures for personality and psychological research. Northwestern University. https://cran. r-project.org/web/packages/psych/

Rogers, M. E., Creed, P. A., \& Glendon, A. I. (2008). The role of personality in adolescent career planning and exploration: A social cognitive perspective. Journal of Vocational Behavior, 73(1), 132-142. https://doi.org/10.1016/ j.jvb.2008.02.002

Rosseel, Y. (2012). Lavaan: An R package for structural equation modeling. Journal of Statistical Software, 48(2), 1-36. http://www.jstatsoft.org/v48/i02/paper

Santos, L. A. B. (2013). Formação em contexto militar. Revista Militar, 2533/2534, 143-166. https://www.revistamilitar. $\mathrm{pt} /$ artigo/804

Savickas, M. L. (1999). The transition from school to work: A developmental perspective. The Career Development Quarterly, 47(4), 326-336. https://doi.org/10.1002/j.2161-0045.1999.tb00741.x

Savickas, M. L. (2013). Career construction theory and practice. In S. D. Brown \& R. W. Lent (Eds.), Career development and counseling: Putting theory and research to work (pp. 147-183). John Wiley \& Sons.

Savickas, M. L., \& Porfeli, E. J. (2012). Career adapt-abilities scale: Construction, reliability, and measurement equivalence across 13 countries. Journal of Vocational Behavior, 80(3), 661-673. https://doi.org/10.1016/ j.jvb.2012.01.011

Sbicigo, J. B., Teixeira, M. A. P., Dias, A. C. G., \& Dell'Aglio, D. D. (2012). Propriedades psicométricas da Escala de Autoeficácia Geral Percebida (EAGP). Psico, 43(2), 139- 146. http://revistaseletronicas.pucrs.br/ojs/index.php/ revistapsico/article/view/11691/8037

Schaufeli, W. .B., Salanova, M., González-Romá, V., \& Bakker, A. B. (2002). The measurement of engagement and burnout: A confirmatory factor analytic approach. Journal of Happiness Studies, 3, 71-92. https://doi.org/ 10.1023/A:1015630930326

Seibert, S. E., Crant, J. M., \& Kraimer, M. L. (1999). Proactive personality and career success. Journal of Applied Psychology, 84(3), 416-417. https://homepages.se.edu/cvonbergen/files/2013/01/Proactive-Personality-andCareer-Success.pdf

Shafer, S. (2010). Professional development to promote employee engagement: A multi-case study [Dissertação de mestrado não publicada]. Clemson University.

Sinha, R. (2020). Career development: An enabler for job satisfaction. In J. Fahed-Sreih (Ed.), Career development and job satisfaction. Intechopen. https://doi.org/10.5772/intechopen.91683

Spurk, D., Abele, A. E., \& Volmer, J. (2011). The career satisfaction scale: Longitudinal measurement invariance and latent growth analysis. Journal of Occupational and Organizational Psychology, 84(2), 315-326. https://doi. org/10.1111/j.2044-8325.2011.02028.x

Stumpf, S. A., Colarelli, S. M., \& Hartman, K. (1983). Development of the Career Exploration Survey (CES). Journal of Vocational Behavior, 22(2), 191-226. https://doi.org/10.1016/0001-8791(83)90028-3

Uhl-Bien, M., Schermerhorn Jr., J. R., \& Osborn, R. N. (2016). Organizational behavior (13th ed.). Wiley.

Wolff, H. G., \& Moser, K. (2009). Effects of networking on career success: A longitudinal study. Journal of Applied Psychology, 94(1), 196-206. https://doi.org/10.1037/a0013350

Wong, S. C., Rasdi, R. M., Samah, B. A., \& Wahat, N. W. A. (2017). Promoting protean career through employability culture and mentoring: Career strategies as moderator. European Journal of Training and Development, 41(3), 277-302. https:// doi.org/10.1108/EJTD-08-2016-0060

Yap, M., Cukier, W., Holmes, M. R., \& Hannan, C.-A. (2010). Career satisfaction: A look behind the races. Industrial Relations, 65(4), 2010, 584-608. https://doi.org/10.7202/045587ar 
Zhou, M. (2015). A revisit of general self-efficacy scale: Uni- or multi-dimensional? Current Psychology, 35(3), 427-436. https://doi.org/10.1007/s12144-015-9311-4

Zikic, J., \& Hall, D. T. (2009). Toward a more complex view of career exploration. The Career Development Quarterly, 58(2), 181-191. https://doi.org/10.1002/j.2161-0045.2009.tb00055.x

Alexandra de Oliveira Rodrigues-Marçulo

Graduada em Psicologia pela Universidade Federal do Rio de Janeiro (UFRJ), Rio de Janeiro - RJ. Mestra e Doutora em Psicologia: Cognição, Organizações e Trabalho pela Universidade Salgado de Oliveira (Universo), Niterói - RJ. MBA em Gestão de Pessoas pela Fundação de Apoio ao Cefet (Funcefet). Especialista em Planejamento, Implementação e Gestão da Educação a Distância (Pigead) pela Universidade Federal Fluminense (UFF), Niterói - RJ. Especialista em Formação de Psicoterapeutas pelo Centro de Psicologia da Pessoa (CPP), Rio de Janeiro - RJ. Desenvolve estudos na Área de Psicologia Social, do Trabalho e das Organizações.

E-mail: alexandra.psique@gmail.com

(1) https://orcid.org/0000-0001-6389-859X

\section{Maria Cristina Ferreira}

Graduada em Psicologia pela Universidade do Estado do Rio de Janeiro (Uerj), Rio de Janeiro - RJ. Mestra e Doutora em Psicologia, na área de Psicologia Cognitiva, pela Fundação Getúlio Vargas (FGV), Rio de Janeiro - RJ. Realizou estágio pós-doutoral naVictoria University atWellington, Nova Zelândia. Atua principalmente nos seguintes temas: comportamento organizacional positivo, psicologia transcultural e medidas psicológicas. Membro do Comitê de Assessoramento de Psicologia do CNPq.

E-mail: mcris@centroin.com.br

(1) http://orcid.org/0000-0003-0752-6710

\section{Larissa Maria David Gabardo-Martins}

Graduada, Mestra e Doutora em Psicologia pela Universidade Salgado de Oliveira (Universo), Niterói - RJ, Brasil. Pós-graduada em Apoio Matricial, com ênfase no Núcleo de Apoio à Saúde da Família, no qual trabalhou por 5 anos. Tem experiência em Psicologia Clínica, com ênfase em atendimentos infantis. Na área acadêmica, atua principalmente nos seguintes temas: bem-estar, interface trabalho-família, psicometria, validação de escala, métodos estatísticos, psicologia e saúde e psicologia e educação.

E-mail: laragabardo@yahoo.com.br

(1) https://orcid.org/0000-0003-1356-8087

Endereço para envio de correspondência:

Instituto Federal de Roraima, Diretoria de Ensino de Graduação (DEG). Av. Glaycon de Paiva, 2496, Pricumã. CEP: 69303-340. Boa Vista - RR. Brasil.

Recebido 01/12/2019

Aceito 04/03/2021

Received 12/01/2019

Approved 03/04/2021

Recibido 01/12/2019

Aceptado 04/03/2021 
Rodrigues-Marçulo, A. de O., Ferreira, M. C., \& Gabardo-Martins, L. M. D. (2022). Validação da Escala de Engajamento na carreira.

Como citar: Rodrigues-Marçulo, A. de O., Ferreira, M. C., \& Gabardo-Martins, L. M. D. (2022). Evidências de validade da Escala de Engajamento na carreira no contexto militar. Psicologia: Ciência e Profissão, 42, 1-15. https://doi.org/10.1590/1982-3703003231619

How to cite: Rodrigues-Marçulo, A. de O., Ferreira, M. C., \& Gabardo-Martins, L. M. D. (2022). Validity evidence of the Career Engagement Scale in the military context. Psicologia: Ciência e Profissão, 42, 1-15. https://doi.org/10.1590/1982-3703003231619

Cómo citar: Rodrigues-Marçulo, A. de O., Ferreira, M. C., \& Gabardo-Martins, L. M. D. (2022). Evidencias de validez de la Escala de Compromiso Profesional en el contexto militar. Psicologia: Ciência e Profissão, 42, 1-15. https://doi.org/10.1590/1982-3703003231619 